\title{
Investigation of multiphoton-induced fluorescence from solutions of 5-hydroxytryptophan
}

\author{
Roger H.Bisby*a, Morfakis Arvanitidis ${ }^{a}$, Stanley W.Botchway ${ }^{\mathrm{b}}$, Ian P.Clark ${ }^{\mathrm{b}}$, Anthony \\ W.Parker ${ }^{b}$ and Darren Tobin ${ }^{a}$
}

*Author for correspondence; email address:- r.h.bisby@salford.ac.uk

${ }^{a}$ Biosciences Research Institute, University of Salford, Salford M5 4WT, UK

${ }^{\mathrm{b}}$ Lasers for Science Facility, Rutherford Appleton Laboratory, Chilton, Didcot, Oxon OX11 OQX, UK

\begin{abstract}
It is reported (Shear et al, Photochem.Photobiol. $\underline{65}, 931$ (1997) ) that multiphoton near infrared excitation of 5-hydroxytryptophan results in a transient product with green fluorescence. Visible fluorescence from multiphoton excitation enables detection of 5-hydroxytryptophan with extremely high sensitivity and also has potential applications in imaging of biological systems and investigation of protein dynamics. The characteristic fluorescence at $500 \mathrm{~nm}$ has now also been observed on one-photon photolysis of solutions at $308 \mathrm{~nm}$, followed by excitation of fluorescence at $430 \mathrm{~nm}$. Fluorescence was observed in aerated and deaerated solutions and in the presence of ascorbate. Enhancement of fluorescence was observed on addition of ethanol. Transient absorption experiments with 308 $\mathrm{nm}$ photolysis showed the formation of three transient species. In the presence of ascorbate the radical formed by photoionisation was removed, revealing a long-lived species (. $>1 \mathrm{~ms})$ with a similar absorption spectrum, which is ascribed to the fluorescing species. Fluorescence induced by multiphoton excitation had a lifetime of $910 \pm 10$ ps and was also unaffected by ascorbate. In the presence of organic solvents there was an increase in fluorescence lifetime, but a decrease in overall fluorescence intensity. The fluorescence intensity and fluorescence lifetime both decreased in acidic solution $(\mathrm{pH}<3)$. It is suggested that the fluorescence originates not from the 5-indoxyl radical, but from a transient product formed by intramolecular rearrangement.
\end{abstract}




\section{INTRODUCTION}

Multiphoton excitation of chromophores in biological imaging ${ }^{1,2}$ and quantitative fluorescence measurements ${ }^{3}$ reduces background luminescence and has the potential to increase sensitivity and selectivity. Multiphoton excitation is usually used to excite the same fluorescent species as normally obtained under conventional one photon ultraviolet or visible illumination. In the case of 5-hydroxytryptophan $(5 \mathrm{HT})$, multiphoton excitation with the output of a Ti-sapphire laser in the region of 720 to $800 \mathrm{~nm}$ gives rise to an additional luminescent signal with a maximum at about $500 \mathrm{~nm}$ (described as "hyperluminescence"), compared with the normal UV-excited fluorescence of $5 \mathrm{HT}$ at around $340 \mathrm{~nm}^{4}$. This visible has allowed as little as $70 \mathrm{zmol}$ of $5 \mathrm{HT}$ to be detected ${ }^{5}$. In addition, the visible fluorescence from multiphoton excitation of proteins containing $5 \mathrm{HT}$ in place of tryptophan itself allows them to be imaged separately from other proteins ${ }^{6}$. The fluorescence lifetime of multiphoton-induced hyperluminescence from $5 \mathrm{HT}$ in aqueous solutions has been determined to be about $0.8 \mathrm{~ns}$ and the intensity of hyperluminescence shown to depend on oxygen concentration and the presence of buffer solutes ${ }^{7}$.

The origins of the visible fluorescence of $5 \mathrm{HT}$ are unknown. Webb and co-workers have shown that the emission arises from an overall 6-photon process with $830 \mathrm{~nm}$ photons, and may be resolved into a 4-photon excitation of $5 \mathrm{HT}$ to the transient fluorescing intermediate, and a 2-photon excitation of fluorescence ${ }^{4}$. Luminescence in the region of $550 \mathrm{~nm}$ has also been reported from 5 -hydroxytryptamine in acidic solutions ${ }^{8}$. Shear et al ${ }^{4}$ suggest that multiphoton-induced hyperluminescence from $5 \mathrm{HT}$ might arise from the 5 -indoxyl radical, known to be formed by photoionisation of $5 \mathrm{HT}$ by $248 \mathrm{~nm}$ laser flash photolysis ${ }^{9}$, and which has also been studied by pulse radiolysis ${ }^{10-13}$. In neutral solutions the 5 -indoxyl radical has an absorption maximum at $410-420 \mathrm{~nm}^{10,13}$, consistent with two-photon excitation of hyperluminescence by ca. $800 \mathrm{~nm}$ laser pulses.

In the present experiments we have attempted to characterise the fluorescence from photoexcited $5 \mathrm{HT}$ using both one-photon and multiphoton excitation. The results suggest that the fluorescence does not arise from the 5-indoxyl radical.

\section{MATERIALS AND METHODS}

5-Hydroxytryptophan and other chemicals were obtained from Sigma-Aldrich and used as received. Solutions were prepared in water purified by ion-exchange and filtration in a Barnstead unit, and buffered to the indicated $\mathrm{pH}$ values with phosphate or $\mathrm{HCl}$.

The fluorescence induced by one-photon excitation (OPE) of 5-HT was measured using a pump-probe technique with the same apparatus as previously employed in nanosecond timeresolved resonance Raman experiments ${ }^{14}$. Photochemically-generated transients in $5 \mathrm{HT}$ 
solutions were produced by a $308 \mathrm{~nm}$ pump pulse (ca. $1 \mathrm{~mJ}, 10 \mathrm{~ns}$ ) from a $\mathrm{XeCl}$ laser (Lumonics). This was followed after a set time interval by a $430 \mathrm{~nm}$ probe laser pulse (ca. 1 $\mathrm{mJ}, 5 \mathrm{~ns}$ ) from a dye laser (Continuum YAG-pumped Sirah). Luminescence excited by both lasers was dispersed by a Spex Triplemate spectrograph and detected by a Princeton Instruments CCD. Spectra were averaged over 200 laser pulses. Due to the low dispersion of the spectrograph, fluorescence spectra we recorded over ca. $25 \mathrm{~nm}$ ranges and normalised by reference to fluorescence from a solution of coumarin 102 in methanol excited only by the $430 \mathrm{~nm}$ laser. A corrected spectrum of the same coumarin 102 solution was recorded in a Spex Fluoromax spectrofluorimeter, employing the manufacturer's correction curve, and used to correct the $5 \mathrm{HT}$ luminescent spectrum. For the measurements, the solutions were recirculated through a quartz tube (i.d. $2 \mathrm{~mm}$ ) at a rate sufficient to ensure the sample was completely replenished between laser pulses. Laser flash photolysis experiments used only the $\mathrm{XeCl}$ laser and conventional detection of transient species by time-resolved absorption spectroscopy.

For the multiphoton excitation (MPE) experiments the output of a titanium sapphire laser (Spectra-Physics) operating at $710-820 \mathrm{~nm}, 80 \mathrm{MHz}$ with $120 \mathrm{fs}$ pulse width was focussed at a microscope stage (Olympus IMT-2) and into the sample using a $x 40$ objective, numerical aperture 0.85 . Samples were illuminated with a laser power of $10 \mathrm{~mW}$ for determination of emission spectra and fluorescence lifetimes. The same objective collected the hyperluminescence that was re-imaged at the slit of a monochromator (IBH Ltd). Emission was detected with a single-photon counting micro-channel plate photomultiplier (Hamamatsu R3809-U) with a response time of $35 \mathrm{ps}$. Lifetime measurements were performed using a Becker and Hickl GmbH time-correlated single photon counting PCI module (SPC-700) with a time resolution of $830 \mathrm{fs} / \mathrm{channel}$.

Pulse radiolysis experiments were performed using the linac (10 MeV, 500 ns electron pulses) at the Facility for Free Radical Research at the Daresbury Laboratory (FFRR@DL). Transient species were detected by time-resolved absorption pectroscopy in the UV/visible region.

\section{RESULTS AND DISCUSSION}

\section{1) Fluorescence from one-photon excitation (OPE)}

At neutral $\mathrm{pH}, 5 \mathrm{HT}$ has a significant absorption at $<320 \mathrm{~nm}$, and may therefore be excited by the $308 \mathrm{~nm}$ output of a $\mathrm{XeCl}$ excimer laser. Fluorescence spectra recorded from onephoton excitation of a deaerated solution of $5 \mathrm{HT}$ at $\mathrm{pH} 7$ are shown in Figure 1. The spectra are corrected for detector response and spectrometer efficiency by comparison with a spectrum of coumarin 102 in methanol recorded under identical conditions, itself normalised to a the corrected spectrum of coumarin 102 recorded using a Spex Fluoromax fluorimeter 
with the manufacturer's correction response curve. The sample was initially illuminated with a $308 \mathrm{~nm}$ (10 ns) laser pulse (the 'pump' pulse) to generate the photochemical intermediate(s) from $5 \mathrm{HT}$. Figure 1 shows that in the pump-only experiment, curve $\mathrm{A}$, fluorescence is generated with a peak at ca. $500 \mathrm{~nm}$. The additional fluorescence at $<450 \mathrm{~nm}$ is believed to be the red tail of the of the normal fluorescence of $5 \mathrm{HT}$, which has a peak at $\sim 340 \mathrm{~nm}$. An increase in luminescence intensity was observed when the sample was subsequently illuminated after a short time delay with a second laser pulse at $430 \mathrm{~nm}$ (the 'probe' pulse). At a time delay of only $10 \mathrm{~ns}$ between pump and probe laser pulses, curve $B$, slightly increased fluorescence is observed to result from the $430 \mathrm{~nm}$ excitation. Increasing the time delay to 1 $\mathrm{s}$ (curve $\mathrm{C}$ ) and $100 \mathrm{~s}$ (curve $\mathrm{D}$ ) results in further increase in the $500 \mathrm{~nm}$ fluorescence. The inset to Figure 1 shows that the increase in fluorescence intensity at $500 \mathrm{~nm}$ with delay between pump and probe pulses occurs with a time constant ( ) of 3.5 s. Curve E in Figure 1 shows the emission resulting when the pump laser is turned off and the sample receives only the $430 \mathrm{~nm}$ pulse. Under these conditions only a weak and relatively much narrower peak at $504 \mathrm{~nm}$ is observed. This peak clearly corresponds to the Raman band of the water solvent ${ }^{15}$ at 3400 to $3450 \mathrm{~cm}^{-1}$.

Table 1 shows the fluorescence intensities at $500 \mathrm{~nm}$ under a range of conditions. With the sample irradiated only with the pump laser $(308 \mathrm{~nm})$ pulse there was no difference between solutions saturated with oxygen or deoxygenated with argon. A similar situation was found with the pump $(308 \mathrm{~nm})$ laser pulse followed by a probe $(430 \mathrm{~nm})$ laser pulse delayed by only $10 \mathrm{~ns}$. However the presence of oxygen decreased the fluorescence intensity by $15 \%$ when the delay between pump and probe laser pulses was increased to $200 \mathrm{~s}$. The presence of ascorbate in the 5-hydroxytryptophan solutions at concentrations up to $4 \mathrm{mmol} \mathrm{dm}^{-3}$ was found to have no effect on the fluorescence intensity. The intensity of the $500 \mathrm{~nm}$ fluorescence was also measured in an ethanol/water (50:50 v/v) mixture with a pump to probe delay of $50 \mathrm{~s}$ and found to be 1.9 times higher than in water alone.

\section{Fluorescence from multiphoton excitation (MPE)}

a) Excitation and emission spectra. Spectra from multiphoton excitation of $5 \mathrm{HT}$ were recorded in a number of solvents. The excitation spectrum of an aqueous solution of $5 \mathrm{HT}(10 \mathrm{mmol} \mathrm{dm}-$ ${ }^{3}$ ) buffered to $\mathrm{pH} 7.0$ with phosphate $\left(50 \mathrm{mmol} \mathrm{dm}^{-3}\right)$ shown in Figure 2 indicates a peak at $740 \mathrm{~nm}$. This represents an unresolved superimposition of the spectra for photochemical activation of $5 \mathrm{HT}$ and for excitation of fluorescence from the intermediate. Without our being able to modulate power in the laser as described by Shear et $\mathrm{al}^{4}$, we are unable to resolve separate excitation spectra for the two processes or ascertain relative contributions. The emission spectra on multiphoton excitation at 773 to $785 \mathrm{~nm}$ for $5 \mathrm{HT}$ in aqueous buffer and in ethanol are shown in Figure 3. The spectra have been corrected by reference to the emission spectrum of coumarin 102 in the same way as described for the nanosecond results. In neutral aqueous buffer the emission spectrum peaks at $500 \mathrm{~nm}$ and is very similar to that 
measured in the nanosecond pump-probe experiment. The slight discrepancy at long wavelengths may be due to deficiencies in the correction. The fast photomultiplier used for the detection of the multiphoton excited fluorescence has very poor red response, compared with the virtually flat response over the wavelength region of interest of the CCD detector used for the nanosecond measurements. The inset to Figure 3 shows a log-log plot of emission intensity versus $5 \mathrm{HT}$ concentration for multiphoton excitation in neutral aqueous solution. The slope of this plot is $1.31 \pm 0.05$. In ethanol the emission spectrum of $5 \mathrm{HT}$ on multiphoton excitation is slightly blue shifted to $490 \mathrm{~nm}$ compared with the spectrum measured for $5 \mathrm{HT}$ in aqueous solution.

b) Fluorescence lifetimes In buffered solution at $\mathrm{pH} 7$, the fluorescence of the $500 \mathrm{~nm}$ emission from $5 \mathrm{HT}$ on multiphoton excitation between 740 and $780 \mathrm{~nm}$ was found to decay as a single exponential with a lifetime of $910 \pm 10 \mathrm{ps} \mathrm{(.}$ (.$\tilde{s}$. This is in good agreement with the value of $\approx 0.8 \mathrm{~ns}$ obtained by Gostkowski et al ${ }^{7}$. Figure 4 shows that the fluorescence lifetime remains constant with this value between $\mathrm{pH} 5$ and $\mathrm{pH} 8.5$, but is reduced in acidic solutions with the fluorescence intensity showing a corresponding decrease. The process shows an apparent $\mathrm{pK}_{\mathrm{a}}$ of about 1.5. The inset to Figure 4 shows a Stern-Volmer analysis of the lifetime data as if the excited state was quenched in a dynamic process by protons in acid solution. This leads to a value of $(9.1 \pm 1.1) \times 10^{10} \mathrm{dm}^{3} \mathrm{~mol}^{-1} \mathrm{~s}^{-1}$ for the quenching constant of the excited state by $\mathrm{H}^{+}$.

The fluorescence lifetime of the $5 \mathrm{HT}$ measured with multiphoton excitation at $740 \mathrm{~nm}$ was found to be solvent dependent as shown in the inset to Figure 2. In neutral buffered $D_{2} \mathrm{O}$ solution the lifetime was found to increase to $1.76 \mathrm{~ns}$ compared with $0.91 \mathrm{~ns}$ in $\mathrm{H}_{2} \mathrm{O}$ solution. The lifetime also increased in non-aqueous solutions. Figure 5 shows that in solutions containing $60 \%$ and $90 \%$ ethanol (remainder $\mathrm{H}_{2} \mathrm{O}$ ) the fluorescence lifetime increased from $0.94 \mathrm{~ns}$ in unbuffered $\mathrm{H}_{2} \mathrm{O}$ to $2.25 \mathrm{~ns}$ and $3.1 \mathrm{~ns}$ respectively. In acetonitrile $(90 \% \mathrm{v} / \mathrm{v})$ the lifetime of $3.05 \mathrm{~ns}$ was also increased relative to that in water. Despite these increases in fluorescence lifetimes, the integrated emission intensity in non-aqueous solvents was considerably lower than in water.

The lifetimes and intensities of 5-hydroxytryptophan fluorescence on multiphoton excitation were also measured in solution containing acrylamide (up to $100 \mathrm{mmol} \mathrm{dm}^{-3}$ ) and ascorbate (up to $20 \mathrm{mmol} \mathrm{dm}^{-3}$ ). With both solutes there was no significant quenching of fluorescence intensity or reduction in fluorescence lifetime.

\section{Laser flash photolysis}

Laser flash photolysis with a $308 \mathrm{~nm}$ laser pulse was used to investigate the species formed on photoexcitation. The results are shown in Figure 6. In deaerated solution a short-lived transient $\left(\begin{array}{ll}\sim & \mathrm{s}\end{array}\right)$ is observed which has increasing absorbance towards the red end of the 
spectral range investigated and which is quenched in the presence of air. This is clearly the hydrated electron and indicates that, at least in part, photoexciation leads to photoionisation. The data in Figure 6 for air-saturated solutions shows the formation of another species absorbing at $\sim 360 \mathrm{~nm}$ which decays with a lifetime of about $5 \mathrm{~s}$. The remaining spectrum at $100 \mathrm{~s}$ shows a peak at $410-420 \mathrm{~nm}$. This is very similar to the spectrum of the 5 -indoxyl radical from $5 \mathrm{HT}$ at neutral $\mathrm{pH}$ observed by pulse radiolysis ${ }^{10,13}$ and confirmed by us in our own pulse radiolysis experiments on oxidation of $5 \mathrm{HT}$ by the $\mathrm{Br}_{2}{ }^{-\cdot}$ radical at $\mathrm{pH} 7$ (results not shown). In the laser flash photolysis experiments it was found that in air-saturated solution in the presence of ascorbate there was a partial decay of the 410-420 nm peak (inset to Figure 6) and that with $5 \mathrm{mmol} \mathrm{dm}^{-3}$ ascorbate approximately half the absorption remains after the initial decay. The remaining spectrum also has a peak at about $400 \mathrm{~nm}$, but is relatively more intense at $360 \mathrm{~nm}$ where the ascorbate radical absorbs ${ }^{16}$. A plot of the first order rate for the partial decay of the $410 \mathrm{~nm}$ transient produced by laser flash photolysis versus ascorbate concentration (Figure 7) gives a second order rate constant of $(7.1 \pm 0.4) \times 10^{7} \mathrm{dm}^{3} \mathrm{~mol}^{-1} \mathrm{~s}^{-1}$ for the reaction of ascorbate. In a corresponding pulse radiolysis experiment the authentic 5indoxyl radical was produced by oxidation of $5 \mathrm{HT}\left(5 \mathrm{mmol} \mathrm{dm}^{-3}\right)$ with $\mathrm{Br}_{2}^{-}$at $\mathrm{pH}$ 7.0. Addition of ascorbate (200-900 mol dm$\left.{ }^{-3}\right)$ resulted in complete loss of the transient absorption at 410 $\mathrm{nm}$ with a second order rate constant of $(11 \pm 1) \times 10^{7} \mathrm{dm}^{3} \mathrm{~mol}^{-1} \mathrm{~s}^{-1}$. Given that determination of second order rate constants are subject to overall uncertainties of $15-20 \%$, the two rate constants measured in the laser flash and pulse radiolysis experiments are essentially the same.

\section{DISCUSSION}

The formation of a transient fluorescent species upon photo-excitation of 5-hydroxytryptophan has been studied by nanosecond laser photolysis and by femtosecond near-infrared laser multiphoton excitation. Very similar fluorescence spectra from an unknown transient product were measured using both methods, suggesting that the same species is produced by one photon at $308 \mathrm{~nm}$ and multiphoton absorption within the sub-picosecond ca. $750 \mathrm{~nm}$ laser pulse. The nanosecond experiments therefore might be used to explore the nature of the transient fluorophore. Nanosecond laser photolysis with $308 \mathrm{~nm}$ laser pulses produces fluorescence with a peak at $500 \mathrm{~nm}$. Since this fluorescence is not observed in the same solutions using a conventional fluorimeter, it suggests that an intense $308 \mathrm{~nm}$ laser pulse may both create a transient intermediate from $5 \mathrm{HT}$ and also excite it to fluoresce. A second laser pulse at $430 \mathrm{~nm}$, delayed by up to $100 \mathrm{~s}$ after the $308 \mathrm{~nm}$ pulse, results in additional fluorescence. This increases in intensity with increasing delay between pump and probe pulse, showing a 3.5 microsecond time constant. No fluorescence was observed on illumination of the sample with the $430 \mathrm{~nm}$ pulse only and without the $308 \mathrm{~nm}$ photolysis laser pulse. The $308 \mathrm{~nm}$ photo-induced species responsible for the $500 \mathrm{~nm}$ fluorescence could be an excited state, radical or covalent product. The results eliminate the excited singlet state as the fluorescent species, the latter being too long lived and actually increasing over a 
microsecond period. Further evidence eliminating the triplet state is seen in the effects of oxygenation (Table 1 ) where only a $15 \%$ reduction is observed $200 \mathrm{~s}$ after the photolysis laser pulse. Assuming a typical second order quenching rate constant of the order of $10^{9} \mathrm{dm}^{3}$ $\mathrm{mol}^{-1} \mathrm{~s}^{-1}$, a triplet state would be expected to be quenched by oxygen (ca. $1 \mathrm{mmol} \mathrm{dm}^{-3}$ in oxygen saturated water) with a half life of about a microsecond.

It is known that the 5-indoxyl radical from one-electron oxidation of 5-HT absorbs at $400-420$ $\mathrm{nm}$ in neutral aqueous solution ${ }^{10,13}$. The radical has shown to be produced by laser flash photolysis at $308 \mathrm{~nm}$ in neutral solution (present work) and at $248 \mathrm{~nm}$ in alkaline solution ${ }^{9}$, and suggested by Shear et al ${ }^{4}$ as possibly the species responsible for the transient fluorescence. Whilst fluorescence from radicals is unusual, there are reports of fluorescence from phenoxyl radicals produced by oxidation of vitamin $\mathrm{E}^{17}$ and 1,3,5-trioxylbenzene derivatives ${ }^{18}$. However, the laser flash photolysis results show that $308 \mathrm{~nm}$ photolysis gives rise to 2 products absorbing at $400-420 \mathrm{~nm}$. One component is eliminated by reduction with ascorbate with a rate constant of ca. $10^{8} \mathrm{dm}^{3} \mathrm{~mol}^{-1} \mathrm{~s}^{-1}$. The one-electron reduction potential $\left(E_{1}\right)$ of the 5 -indoxyl radical from $5 \mathrm{HT}$ has been measured as $208 \mathrm{mV}$ at $\mathrm{pH} 13.5^{11}$ and 550 $\mathrm{mV}$ at $\mathrm{pH} 9.1^{9}$ and is consistent with rapid reduction of the 5-indoxyl radical by ascorbate $\left(\mathrm{E}_{1}\right.$ $(\mathrm{pH} 7)=300 \mathrm{mV})^{11}$. In the pump-probe nanosecond laser experiments it was found that ascorbate at concentrations up to $4 \mathrm{mmol} \mathrm{dm}^{-3}$ had no effect on the fluorescence intensity at $200 \mathrm{~s}$ after the photolysis pulse. Based on the second order rate constant of $10^{8} \mathrm{dm}^{3} \mathrm{~mol}^{-1} \mathrm{~s}^{-}$ ${ }^{1}$, at this concentration of ascorbate the 5-indoxyl radical would have a lifetime of $2.5 \mathrm{~s}$, and so would completely eliminate the radical within $200 \mathrm{~s}$. It was also found that ascorbate had no significant effect on the fluorescence intensity in the multiphoton experiments. These results therefore strongly argue against the 5 -indoxyl radical being the fluorescing species. A further possibility is that the fluorescence originates from a decay product of the 5-indoxyl radical. This is most likely to be a dimerization or disproportionation product, since the radical is observed in the pulse radiolysis experiments to decay by second order kinetics. The likelihood of such products being responsible is also diminished by the ascorbate results. However, the concentration of reactive intermediates produced by the conditions of the nanosecond pump-probe experiments is unknown. It is therefore not possible to compare the time constant of the second order process for radical-radical reaction (typically with a second order rate constant of $10^{8} \mathrm{dm}^{3} \mathrm{~mol}^{-1} \mathrm{~s}^{-1}$ ) with that for reaction with ascorbate.

Recently it has been shown that mercaptoethylamine sharply decreases hyperluminescence from 5-hydroxytryptamine ${ }^{19}$. This is claimed to be supporting evidence for the 5 -indoxyl radical as the species responsible for hyperluminescence. Whilst mercaptoethylamine may be a quencher of some types of radicals and singlet oxygen, in the present case this is unlikely. It is known ${ }^{20}$ that the reduction potential of aliphatic thiyl radicals are ca. $1300 \mathrm{mV}$ at pH 6 . Comparison with the reduction potential for the 5-indoxyl radical indicates that repair of the 5indoxyl radical by mercaptoethylamine is thermodynamically extremely unlikely. Indeed, the 
reverse reaction has been demonstrated in which a thiyl radical causes the rapid oxidation of acetaminophen ${ }^{21}$, a compound structurally related to $5 \mathrm{HT}$ and which has a similar oxidation potential.

A further remaining possibility is that the fluorescent species results from photochemical conversion of the short-lived singlet state to a covalently rearranged product. This would account for the fluorescence species being produced and excited within the $10 \mathrm{~ns}, 308 \mathrm{~nm}$ laser pulse, but does not explain why the additional fluorescence excited by the probe laser pulse at $430 \mathrm{~nm}$ continues to grow in intensity with increasing time delay between the two pulses. Further work is required to study the time course of evolution of the fluorescence species under better-defined conditions, such as in the conventional laser flash photolysis experiment. Additional structural information provided by other detection techniques such as time-resolved resonance Raman spectroscopy might also prove useful.

The $500 \mathrm{~nm}$ hyperluminescence induced by multiphoton infrared absorption has been shown to be an extremely sensitive method for the detection of 5-hydroxytryptophan and related compounds ${ }^{5}$. Other results show that hyperluminescence may be used in the intracellular imaging of proteins in which tryptophanyl residues are replaced by 5-hydroxytryptophan ${ }^{6}$. Intracellular imaging of serotonin by multiphoton excitation of the normal UV emission, but not by hyperluminescence, has also been reported ${ }^{22,23}$. Our picosecond time-resolved studies using such multiphoton excitation indicate that the fluorescence lifetime is invariant over the normal physiological $\mathrm{pH}$ range, but has the interesting property of being sensitive to solvent polarity. In proteins bearing a single tryptophan residue, the fluorescence of a substituted 5HT residue might therefore be used to report on exposure to the aqueous solvent. It is noted that whilst the fluorescence lifetime increases with decreasing solvent polarity, the fluorescence intensity unexpectedly decreases. This might result from the separate effects of solvent on the two step process leading to hyperluminescence. In the nanosecond pump-probe experiment, addition of ethanol leads to an increase in fluorescence intensity as might be expected from the increased lifetime of fluorescence in ethanol on multiphoton excitation. The significantly reduced emission intensity on multiphoton excitation in non-aqueous solvents, including ethanol, therefore suggests that the yield of the fluorescent intermediate produced in the multiphoton experiments is markedly reduced in less polar environments.

\section{ACKNOWLEDGEMENTS}

This work was supported by EPSRC through access to the Laser for Science Facility at the Rutherford Appleton Laboratory and by BBSRC through support of DT. The contribution of the Daresbury Laboratory and the assistance of Drs S.Navaratnam and D.Holder with the pulse radiolysis experiments is gratefully acknowledged. 


\section{REFERENCES}

1. C.Xu, W.Zipfel, J.B.Shear, R.M.Williams and W.W.Webb, Multiphoton fluorescence excitation: new spectral windows for biological nonlinear microscopy, Proc.Natl.Acad,Sci. USA, 1996, 93, 10763-10768.

2. B.R.Masters, P.T.C.So, K.H.Kim, C.Beuhler and E.Gratton, Multiphoton excitation microscopy, confocal microscopy, and spectroscopy of living cells and tissues, Adv.Enzymol., 1999, 307, 513-536.

3. D.J.S.Birch, Multiphoton excited fluorescence spectroscopy of biomolecular systems, Spectrochim. Acta A, 2001, 57, 2313-2336.

4. J.B.S.Shear, C.Xu and W.W.Webb, Multiphoton-excited visible emission by serotonin solutions, Photochem.Photobiol., 1997, 65, 931-936.

5. a) M.L.Gostkowski, J.Wei and J.B.Shear, Measurements of serotonin and related indoles using capillary electrophoresis with multiphoton-induced hyperluminescence, Anal.Biochem., 1998, 260, 244-250. b) M.L.Gostkowski, J.B.McDoniel, J.Wei, T.E.Curey and J.B.Shear, Characterizing spectrally diverse biological chromophore using capillary electrophoresis with multiphoton-excited fluorescence, J.Amer.Chem.Soc., 1998, 120, 18-22.

6. S.W.Botchway, A.W.Parker, I.Barba and K.Brindle, Development of a time-correlated single photon counting multiphoton laser scanning confocal microscope, Central Laser Facility Annual Report, 2000-2001, RAL-TR-2001-030:pp. 170-171.

7. M.L.Gostkowski, T.E.Curey, E.Okerberg, T.J.Kang, D.A.V.Bout and J.B.Shear, Effects of molecular oxygen on multiphoton-excited photochemical analysis of hydroxyindoles, Anal.Chem., 2000, 72, 3821-3825.

8. S.Udenfriend, D.F.Bogdanski and H.Weissbach, Fluorescence characteristics of 5hydroxytryptamine (serotonin), Science, 1955, 122, 972-973.

9. S.V.Jovanovic, S.Steenken and M.G.Simic, One-electron reduction potentials of 5indoxyl radicals, J.Phys.Chem., 1990, 94, 3583-3588.

10. S.V.Jovanovic and M.G.Simic, Tryptophan metabolites as antioxidants, Life Chem.Rep., $1985,3,124-130$. 
11. S.Steenken and P.Neta, One-electron reduction potentials of phenols, hydroxy- and aminophenols and related compounds of biological interest, J.Phys.Chem., 1982, 86, 3661-3667.

12. S.V.Jovanovic, S.Steenken and M.G.Simic, Kinetics and energetics of one-electron transfer reactions involving tryptophan neutral and cation radicals, J.Phys.Chem., 1991, $95,684-687$.

13. A.T.Al-Kawini, P.O'Neill, G.E.Admas, R.B.Cundall, A.Junino and J.Maignan, Characterisation of the intermediates produced on one-electron oxidation of 4-, 5-, 6-, and 7-hydroxyindoles by the azide radical, J.Chem.Soc.Perkin Trans. 2, 1992, 657-661.

14. R.H.Bisby and A.W.Parker, Reactions of triplet duroquinone with -tocopherol and ascorbate: a nanosecond laser flash photolysis and time-resolved resonance Raman investigation, J.Amer.Chem. Soc., 1995, 117, 5664-5670.

15. G.E.Walrafen, Raman spectral studies of water structure, J.Chem.Phys., 1964, 40, 32493256.

16. R.H.Schuler, Oxidation of ascorbate anion by electron transfer to phenoxyl radicals, Radiat.Res., 1977, 69, 417-433.

17. M.C.Depew, M.T.Craw, K.MacCormick and J.K.S.Wan, A CIDEP and fluorescence study of the oxidation of vitamin E: the vitamin E radical cation, Journal of Photochemistry and Photobiology, B: Biology, 1987, 1, 229-239

18. N.Ichinose, S.Tojo and T.Majima, Fluorescence measurement of 3,5-dimethoxyphenol radical cation generated by pulse radiolysis in 1,2-dichloromethane, Chemistry Letters 2000, 1126-1127.

19. M.J.Gordon, E.Okerberg, M.L.Gostkowski and J.B.Shear, Electrophoretic characterization of transient photochemical reaction products, J.Amer.Chem.Soc., 2001, 123, 10780-10781.

20. D.A.Armstrong and P.S.Surdar, Reduction potentials and exchange-reactions of thiyl radicals and disulfide radical anions, J.Phys.Chem., 1987, 91, 6532-6537.

21. R.H.Bisby and N.Tabassum, Properties of the radicals formed by one-electron oxidation of acetaminophen, Biochem.Pharmacol., 1988, 37, 2731-2738. 
22. R.M.Williams, J.B.Shear, W.R.Zipfel, S.Maiti and W.W.Webb, Mucosal mast cell secretion processes imaged using three-photon microscopy of 5-hydroxytryptamine autofluorescence, Biophys.J., 1999, 76, 1835-1846.

23. S.Maiti, J.B.Shear, R.M.Williams, W.R.Zipfel and W.W.Webb, Measuring serotonin distribution in live cells with three-photon excitation, Science, 1997, 275, 530-532. 


\section{FIGURE LEGENDS}

FIGURE 1 Fluorescence spectra recorded from a deaerated aqueous solution of 5-hydroxytryptophan $\left(1 \mathrm{mmol} \mathrm{dm}^{-3}\right)$ buffered to $\mathrm{pH} 7.0$ with phosphate (20 $\left.\mathrm{mmol} \mathrm{dm}^{-3}\right)$. Curve $A$ shows the fluorescence when the solution was excited by a pump laser pulse ( $308 \mathrm{~nm}, 10 \mathrm{~ns}$, ca. $1 \mathrm{~mJ}$ ). Additional excitation by a probe laser pulse (430 nm, $5 \mathrm{~ns}$, ca. $1.5 \mathrm{~mJ}$ ) produced curves $B, C$ and $D$ with pumpto-probe delays of $10 \mathrm{~ns}, 1 \mathrm{~s}$ and $100 \mathrm{~s}$ respectively. Excitation with the 430 $\mathrm{nm}$ probe pulse only resulted in curve E. Spectra have been corrected as described in the text.

Inset:- Effect of pump-to-probe pulse delay on the relative intensity if the fluorescence at $500 \mathrm{~nm}$. The solid curve shows an exponential fit with a time constant of $3.55 \mathrm{~s}$.

FIGURE 2 Multiphoton excitation spectrum of 5-hydroxytryptophan $\left(10 \mathrm{mmol} \mathrm{dm}^{-3}\right.$, emission at $495 \mathrm{~nm}$ ) in water buffered to $\mathrm{pH} 7$ with phosphate $\left(50 \mathrm{mmol} \mathrm{dm}^{-3}\right)$. The inset shows the fluorescence decays (excitation $785 \mathrm{~nm}$, emission $495 \mathrm{~nm}$ ) of $5 \mathrm{HT}$ in $\mathrm{H}_{2} \mathrm{O}$ and $\mathrm{D}_{2} \mathrm{O}$ measured by time-correlated single photon counting. Also shown is the laser profile (impulse response function, IRF) determined by the apparatus. The curves have been offset vertically for clarity.

FIGURE 3 Emission spectrum from 5-hydroxytryptophan $\left(10 \mathrm{mmol} \mathrm{dm}^{-3}\right)$ in phosphate buffer (50 mmol dm ${ }^{-3}, \mathrm{pH}$ 7) on multiphoton excitation at $773 \mathrm{~nm}\left(--\right.$ - $_{\text {- }}$ ). This is compared with the emission spectrum of 5-hydroxytryptophan in $90 \%$ ethanol - 10\% water (v/v) on multiphoton excitation at $770 \mathrm{~nm}($ - - - ) , and the single photon emission spectrum in aqueous solution from the nanosecond experiment in Figure 1 (solid line). The spectra are shown normalised in intensity. Inset:- Effect of 5-hydroxytryptophan concentration on emission intensity $(495 \mathrm{~nm})$ on multiphoton excitation at $773 \mathrm{~nm}$.

FIGURE 4 Effect of $\mathrm{pH}$ on the lifetime of fluorescence $(495 \mathrm{~nm})$ from 5-hydroxytryptophan on multiphoton excitation at $750 \mathrm{~nm}$.

Inset:- Stern-Volmer plot of reciprocal fluorescence lifetime versus proton concentration.

FIGURE 5 Effect of solvent on fluorescence intensity and fluorescence lifetime of 5 -hydroxytryptophan on multiphoton excitation at $770 \mathrm{~nm}$. The non-aqueous solutions contained $10 \% \mathrm{H}_{2} \mathrm{O}(\mathrm{v} / \mathrm{v})$ to assist solubility of 5-hydroxytryptophan. 
FIGURE 6 Transient absorption spectra from laser flash photolysis $(308 \mathrm{~nm})$ of 5-hydroxytryptophan $\left(200 \mathrm{~mol} \mathrm{dm}^{-3}\right)$ in phosphate buffer $\left(20 \mathrm{mmol} \mathrm{dm}^{-3}, \mathrm{pH}\right.$ 7.0):- deaerated, $0.5 \mathrm{~s}$ after the laser pulse ( ); air-saturated measured $1 \mathrm{~s}$ ( ), $4 \mathrm{~s}($ ) and $100 \mathrm{~s}$ ( . after the laser pulse; and in the presence of ascorbate $\left(5 \mathrm{mmol} \mathrm{dm}^{-3}\right) 50 \mathrm{~s}$ after the laser pulse ( ). Inset:- transients measured at $420 \mathrm{~nm}$ in the absence (trace A) and presence of ascorbate (0.5 $\mathrm{mmol} \mathrm{dm}^{-3}$, trace $\left.\mathrm{B}\right)$. The traces are offset for clarity.

FIGURE 7 Second order plot for partial reaction of ascorbate with the transient from laser flash photolysis (308 $\mathrm{nm}$ ) of 5-hydroxytryptophan $\left(200 \mathrm{~mol} \mathrm{dm}^{-3}\right)$ in air saturated phosphate buffer $\left(20 \mathrm{mmol} \mathrm{dm}^{-3}, \mathrm{pH} 7.0\right)$. 
TABLE 1 - Effects of sample illumination and additives on relative fluorescence intensity (arbitrary units) at $500 \mathrm{~nm}$ observed from nanosecond laser pump (308 nm) probe $(430 \mathrm{~nm})$ photolysis of aqueous solutions of 5-hydroxytryptophan (1 $\mathrm{mmol} \mathrm{dm}{ }^{-3}$ ) buffered to $\mathrm{pH} 7.0$ with phosphate $\left(20 \mathrm{mmol} \mathrm{dm}^{-3}\right)$.

\begin{tabular}{|l|c|c|c|}
\hline \hline Lasers and time delay & Gas & Additive & $\begin{array}{c}\text { Relative } \\
\text { Intensity }\end{array}$ \\
\hline \hline Pump Only & Argon & ---------- & 100 \\
\hline & Oxygen & --------- & 104 \\
\hline \hline Pump \& Probe, $10 \mathrm{~ns}$ & Argon & --------- & 151 \\
\hline & Oxygen & ----- & 157 \\
\hline & Argon & Ascorbate $\left(1 \mathrm{mmol} \mathrm{dm}^{-3}\right)$ & 159 \\
\hline \hline Pump \& probe, $200 \mathrm{~s}$ & Argon & ------------ & 323 \\
\hline & Oxygen & ------------ & 276 \\
\hline & Argon & Ascorbate $\left(1 \mathrm{mmol} \mathrm{dm}^{-3}\right)$ & 314 \\
\hline & Argon & Ascorbate $\left(2 \mathrm{mmol} \mathrm{dm}^{-3}\right)$ & 313 \\
\hline & Argon & Ascorbate $\left(4 \mathrm{mmol} \mathrm{dm}^{-3}\right)$ & 313 \\
\hline
\end{tabular}




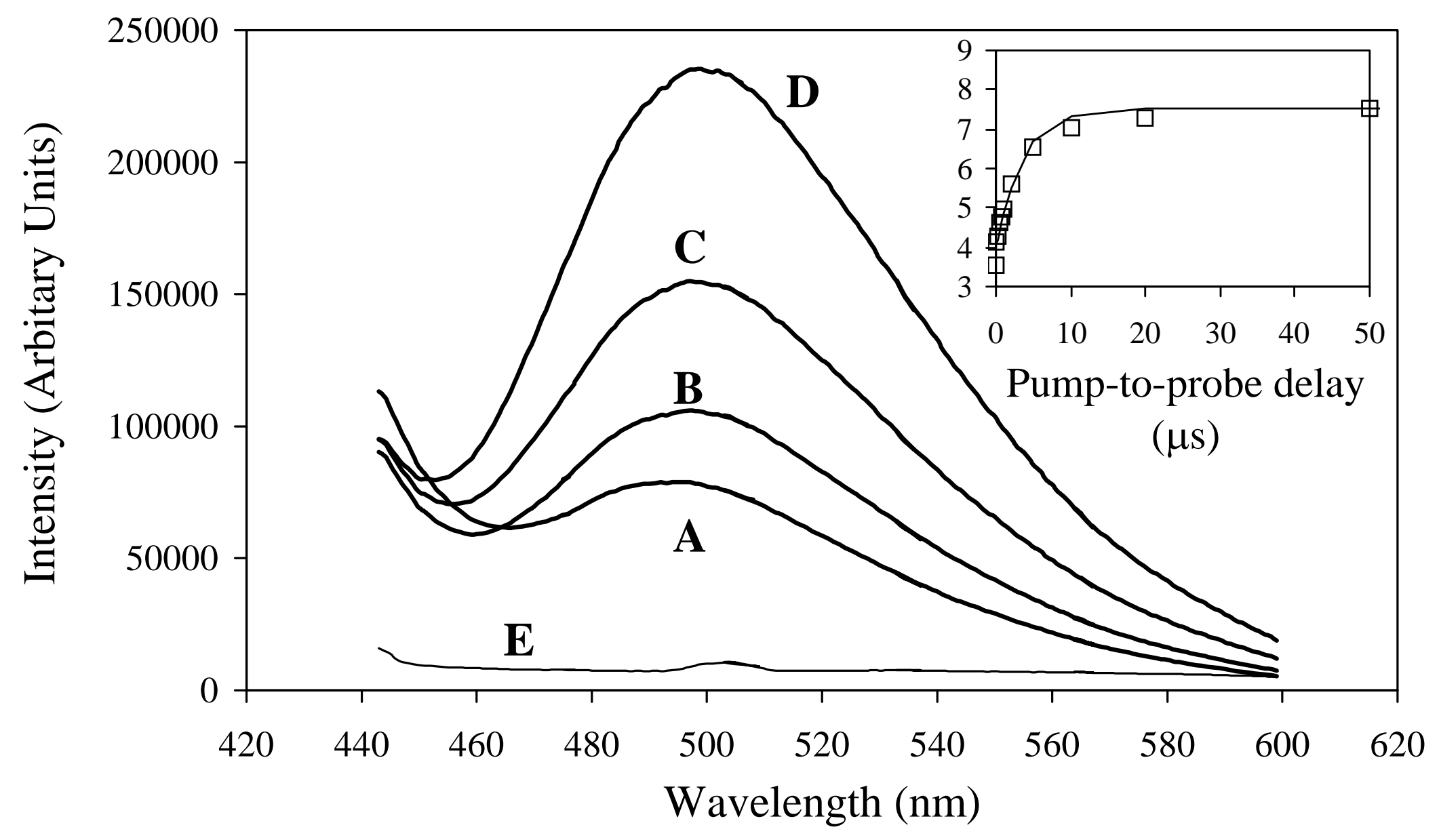

FIGURE 1 


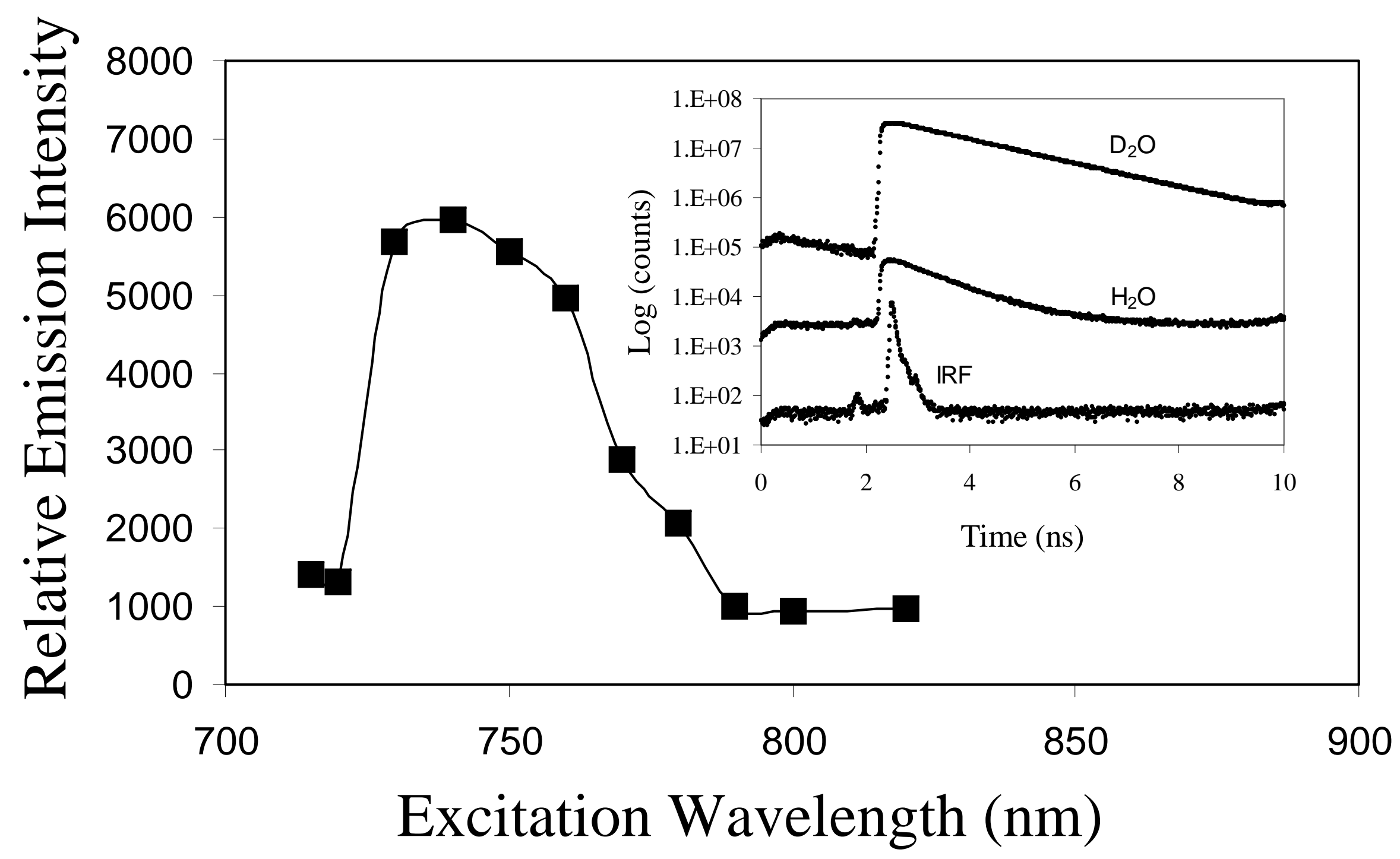

FIGURE 2 


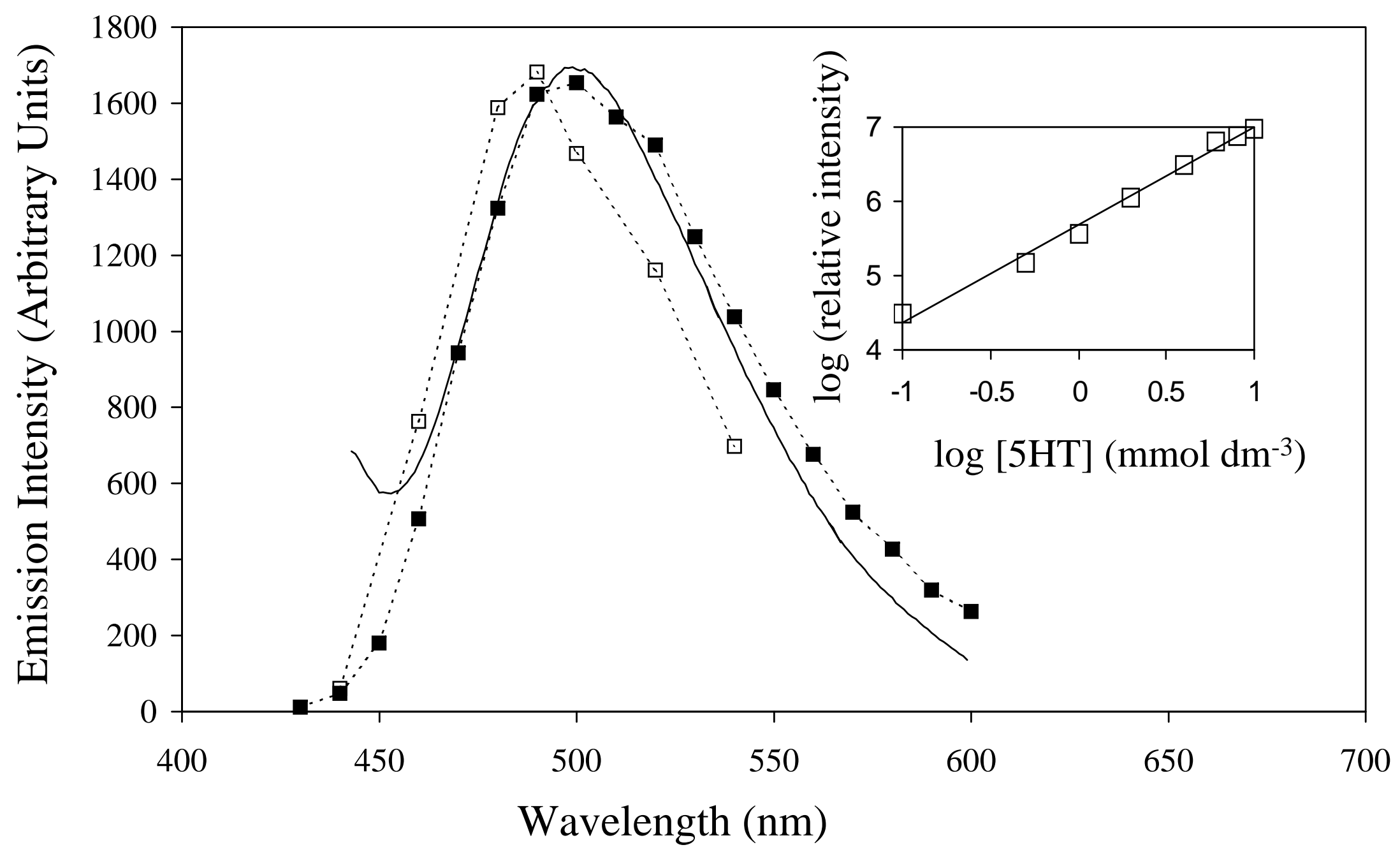

FIGURE 3 


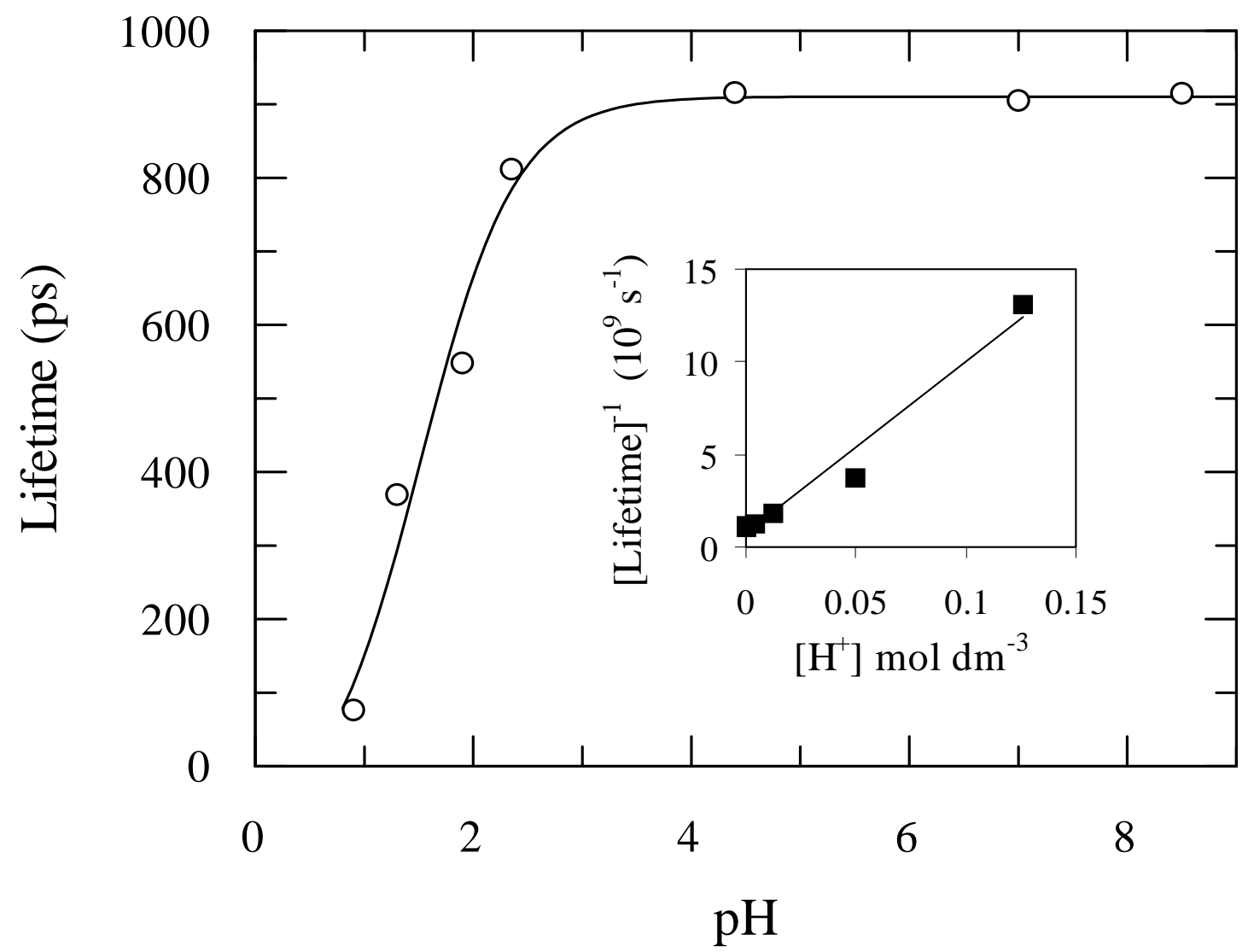

FIGURE 4 


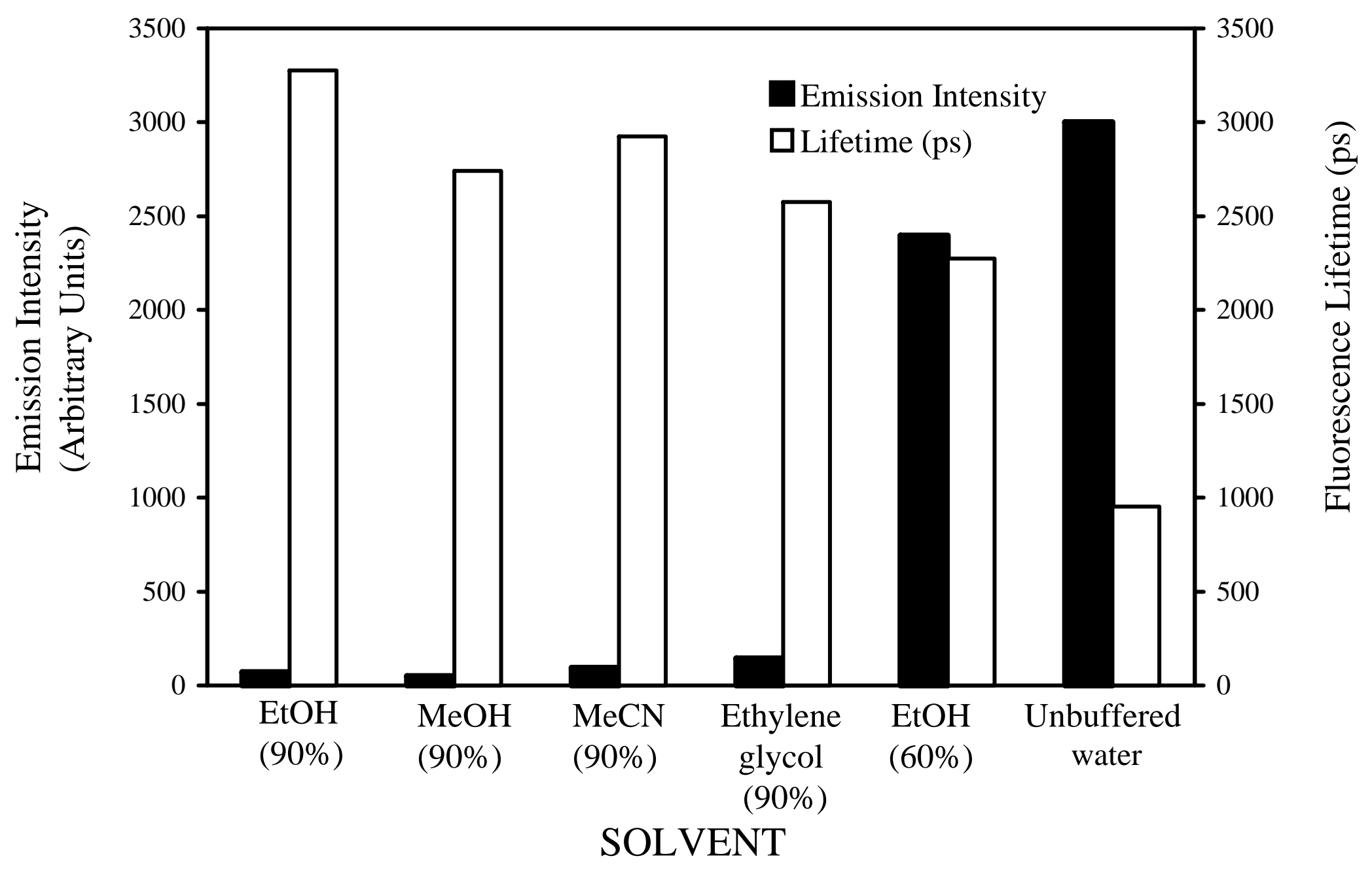

FIGURE 5 


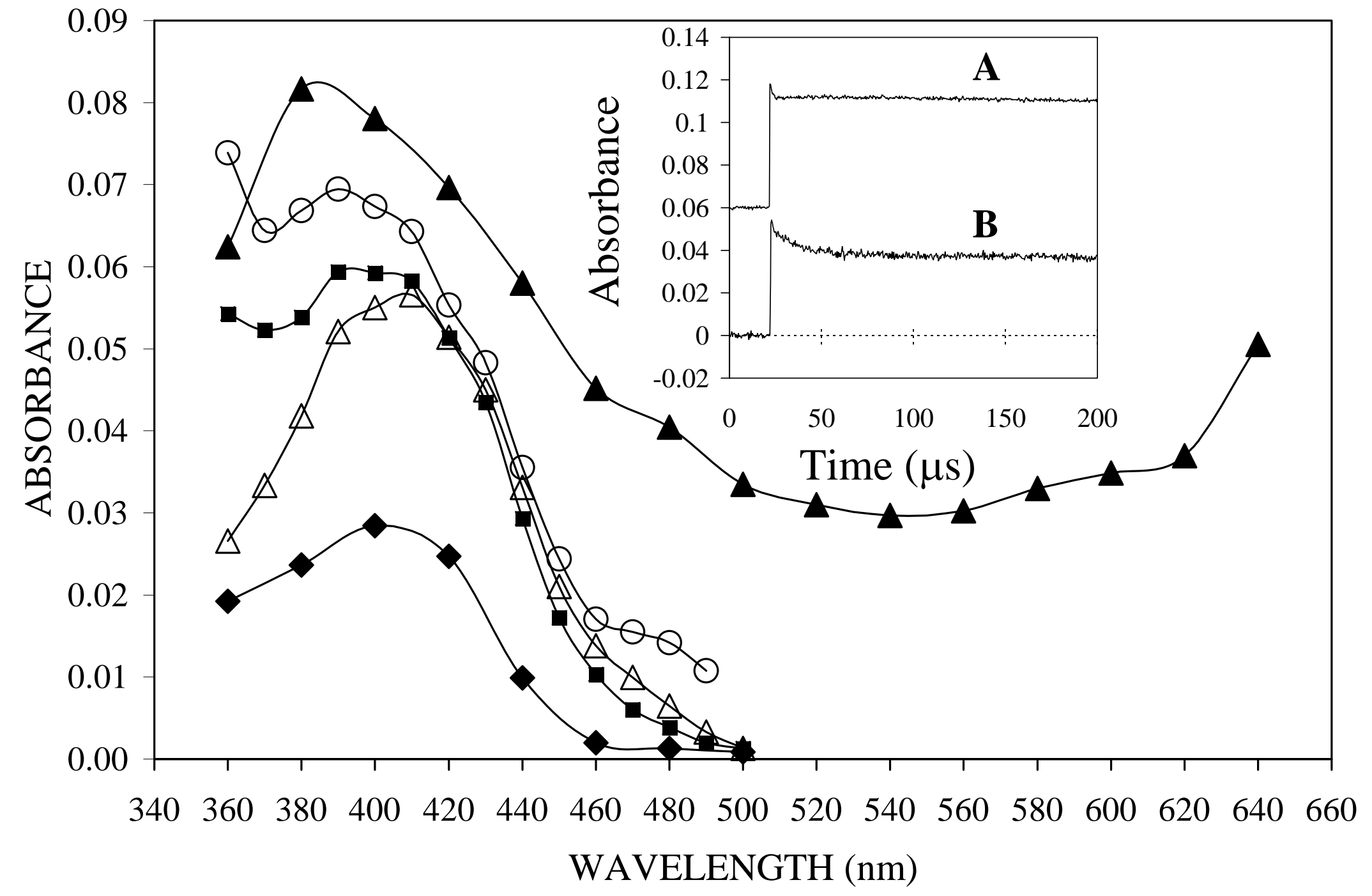

FIGURE 6 


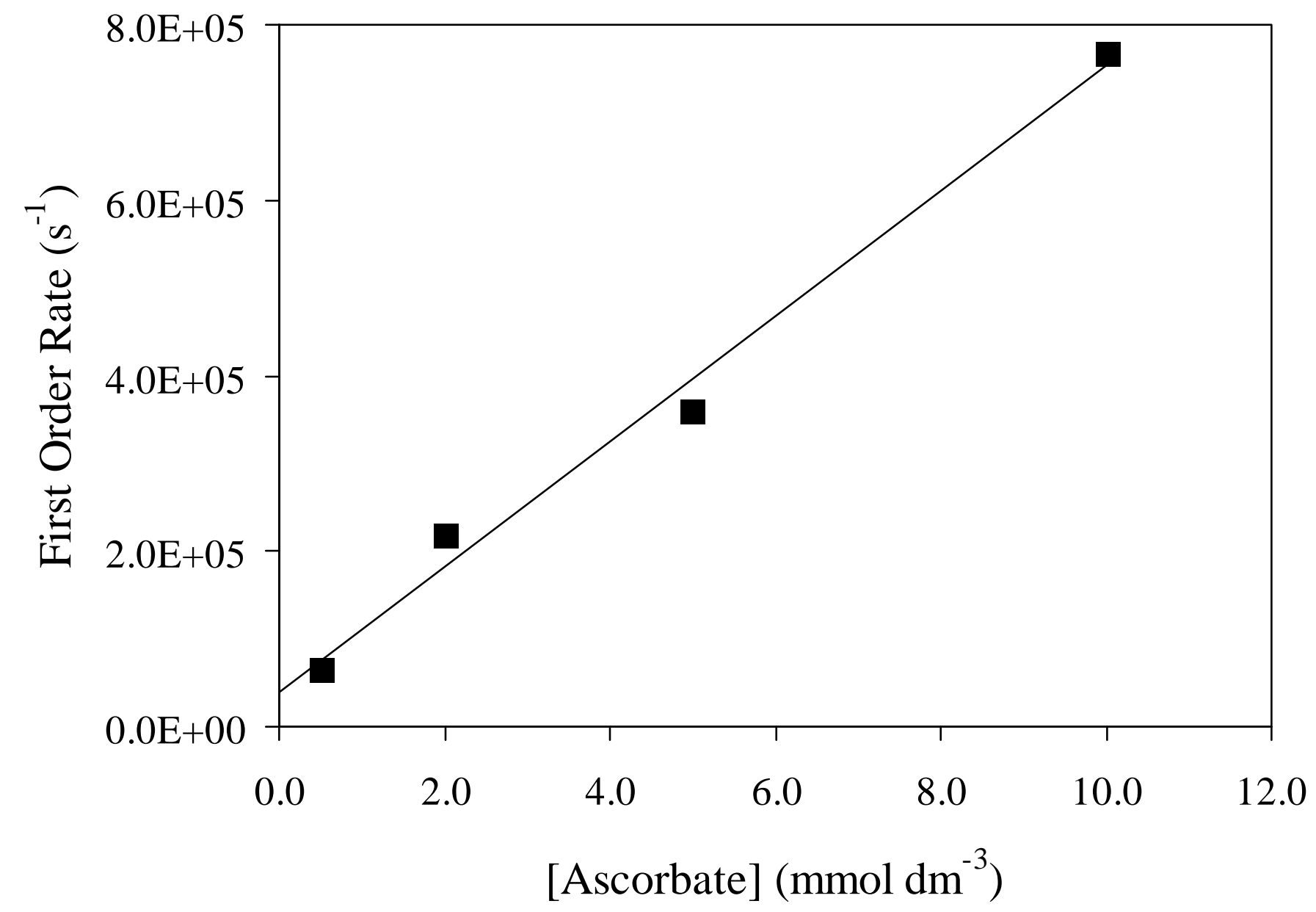

FIGURE 7 